\title{
Role of human serum albumin and oxidative stress in diabetes
}

\begin{abstract}
Changes in human behaviour and lifestyle over the last century have resulted in a dramatic increase in the incidence of diabetes worldwide. Albumin, a major protein in the blood circulation, can undergo increased glycation in diabetes. From recent studies, it has become evident that glycation has important implications for albumin actions and impact on cell functioning. Human serum albumin (HSA) is the most abundant serum protein whose redox modifications modulate its physiological function, as well as serves as bio-marker for oxidative stress. Previous experimental and clinical studies report that oxidative stress plays a major role in the pathogenesis and development of complications of both types of DM. Hypoalbuminemia is the strongest clinical indicator of progression of many human diseases including cancer, diabetes, rheumatic diseases, liver and other diseases. Also, there is currently great interest in the potential contribution of increased oxidative stress to the development of complications in diabetes mellitus. This mini review highlights the association of human serum albumin and oxidative stress in diabetes.
\end{abstract}

Keywords: albumin, oxidative stress, reactive oxygen species, diabetes
Volume 3 Issue I - 2017

\section{Satyam Prakash}

Department of Biochemistry, Janaki Medical College Teaching Hospital, Nepal

\begin{abstract}
Correspondence: Satyam Prakash, Assistant Professor, Department of Biochemistry, Janaki Medical College Teaching, Hospital,Tribhuvan University, Janakpur- 45600, Nepal, Tel +977984I603704, Email sprakashy2424@gmail.com
\end{abstract}

Received: February 12, 2017| Published: May 08, 2017
Abbreviations: AGEs, advanced glycation end-products; ROS, reactive oxygen species; DM, diabetes mellitus; RAGE, receptors of AGEs; HLA, human leucocyte antigen; GDM, gestational diabetes mellitus; MODY, maturity onset diabetes of the young; SMBG, self monitoring blood glucose; HAS, human serum albumin; HMA, human mercaptalbumin; PCO, protein carbonyl; NADPH, nicotinamide adenine dinucleotide phosphate oxidase

\section{Introduction}

Diabetes is an important metabolic disorder which is characterized by hyperglycemia with variable degree of insulin resistance, impaired insulin secretion \& increased glucose levels for Type-I (insulindependent) and Type-II (non-insulin-dependent) diabetes mellitus. ${ }^{1,2}$ Type 1 diabetes mellitus is characterized by chronic insulin deficiency and hyperglycemia due to extensive destruction of insulin-producing beta cells. The autoimmune nature of this process is supported by the presence of a pool of auto antibodies against beta cell antigens. ${ }^{3}$ Nonenzymatic glycation is one of the underlying modification factors that contribute to various alterations of intrinsic protein functions. It is the result of covalent binding of glucose to amino groups of circulating proteins, such as hemoglobin $(\mathrm{HbAlc})$ and albumin, or proteins present in the extracellular matrix (such as collagen and laminin). ${ }^{4}$ Because of its long half-life (about 21 days) compared with other proteins and its high concentrations in the circulatory system, serum albumin is a plasmatic protein that is highly sensitive to glycation. Elevated levels of glycated albumin (two- to threefold increase) in cases of diabetes mellitus can lead to irreversible damage associated with metabolic disorders such as retinopathy, nephropathy, neuropathy and coronary artery disease. ${ }^{5}$ The development of these diabetic complications is attributed to the formation of deleterious and irreversible conjugates called "advanced glycation end-products" (AGEs) during the glycation process. ${ }^{6}$

Elevated blood glucose induces oxidative stress and changes in the cellular redox state. NADPH oxidase has been responsible for the formation of high levels of reactive oxygen species (ROS) in response to high glucose. ${ }^{7,8}$ Elevated levels of oxidized albumin in patients with diabetes mellitus (DM) aging patients with chronic hepatitis-C has been reported. ${ }^{7}$ Hyperglycemia increases the production of free radical and decreases their scavenging by numbers of mechanisms. Diabetes is a major health problem that is usually associated with obesity, together with hyperglycemia and increased advanced glycation end products (AGEs) formation. Elevated AGEs elicit severe downstream consequences via their binding to receptors of AGEs (RAGE). This includes oxidative stress and oxidative modifications of biological compounds together with heightened inflammation. For example, albumin (major circulating protein) undergoes increased glycoxidation with diabetes and may represent an important biomarker for monitoring diabetic pathophysiology. ${ }^{6}$ The main toxic effect of both ROS and AGEs is the induction of abnormal posttranslational modifications of self-antigens and the generation of neo-antigens, thus bypassing immune tolerance and contributing to the development of autoimmune responses. ${ }^{8,9}$ This review overviews the role of albumin and oxidative stress in diabetes.

\section{Diabetes}

Diabetes mellitus (DM) comprises a group of common metabolic disorders that share the phenotype of hyperglycemia. Several distinct types of DM exist and are caused by a complex interaction of genetics, environmental factors and life-style choices. Depending on the etiology of the DM, factor contributing to hyperglycemia may include reduced insulin secretion, decreased glucose utilization and increased glucose production. ${ }^{10} \mathrm{DM}$ is a state of chronic hyperglycemia associated with a high risk of atherosclerosis, renal, nervous system, ocular damage that leads to increase myocardial infarction, stroke and limb amputation. ${ }^{11,12} \mathrm{~A}$ wide spectrum of pathogenic processes is involved in the development of DM which may range from autoimmune destruction of beta cells to the development of insulin resistance. The major risk factors of DM are age, obesity, lack of 
exercise, along with a strong genetic predisposition. A strong Human Leucocyte Antigen (HLA) association with some forms of the disease along with some specific antibodies has also been identified. The pathogenic link between hyperglycemia and the complications of diabetes is unknown. ${ }^{11}$

DM is classified on the basis of the pathogenic process that leads to hyperglycemia, as opposed to earlier criteria such as age of onset or type of therapy. The two broad categories of DM are designated type 1 and type 2. Type 1 has been further subdivided into 1A and 1B. Type 1A DM results from autoimmune beta cell destruction, which leads to insulin deficiency. Individuals with type 1B DM lack immunologic markers indicative of an autoimmune destructive process of the beta cells. However, they develop insulin deficiency by unknown mechanisms. Type $2 \mathrm{DM}$ is a heterogeneous group of disorders characterized by variable degree of insulin resistance, impaired insulin secretion and increased glucose production. Distinct genetic and metabolic defects in insulin action and/or secretion give rise to the common phenotype of hyperglycemia in type $2 \mathrm{DM} .{ }^{10}$ It is frequently associated with permanent and irreversible functional and structural changes in the cells of the body particularly in vascular systems which affect eyes, kidneys, nerves, heart and blood vessels. ${ }^{2,13,14}$

However, the American Diabetes Association, gave other etiologies for DM include specific genetic defects in insulin secretion or action, metabolic abnormalities that impair insulin secretion, mitochondrial abnormalities and a host of conditions that impair glucose tolerance. Maturity onset diabetes of the young (MODY) is a subtype of DM characterized by autosomal dominant inheritance, early onset of hyperglycemia and impairment in insulin secretion. Mutations in the insulin receptor cause a group or rare disorders characterized by severe insulin resistance. ${ }^{13}$ DM can result from pancreatic exocrine disease when the majority of pancreatic islet $(>80 \%)$ are destroyed. Hormones that antagonize the action of insulin can lead to DM. Thus, DM is often a feature of endocrinopathies, such as acromegaly and Cushing's disease. Viral infections have been implicated in pancreatic islet destruction, but are an extremely rare cause of DM. Congenital rubella greatly increases the risk for DM, however, most of these individuals also have immunologic markers indicative of autoimmune beta cell destruction. Another form of DM is gestational diabetes mellitus (GDM), GDM occurs as insulin resistance related to the metabolic changes of late pregnancy increases insulin requirements and may lead to impaired glucose tolerance. GDM occurs in approximately $4 \%$ of pregnancies in the United States; most women revert to normal glucose tolerance post-partum but have a substantial risk (30-60\%) of developing DM later in life. ${ }^{13}$ According to ADA, diabetic nephropathy occurs in $20-30 \%$ of Type-I and Type-II diabetic patients. $^{1,13}$

The two main clinical parameters used for chronic glycaemic control are glycated $\mathrm{HbAlc}$ levels and Self Monitoring Blood Glucose (SMBG) ${ }^{15}$ Measurement of glucose in blood, a short-term indicator, reflects the diabetic status over a 24 -hour period, while the $\mathrm{HbAlc}$ value represents a long-term glycaemic indicator. However, the HbAlc is not always a relevant indicator of glycaemic control in patients with diabetes-associated pathologies that affect erythrocyte lifespan, such as hemolytic or renal anaemia and liver cirrhosis. ${ }^{16,17}$ In such cases, glycated albumin appears to be an alternative marker for glycaemic control: the glycated albumin level is thought to indicate blood glucose status over a short period ( 2 to 4 weeks), while HbA1c reflects the glycaemic state over a longer period ( 2 months). Indeed, numerous studies support the use of glycated albumin levels in the detection of short-term changes in glycaemic control. ${ }^{18-20}$

\section{Human serum albumin}

Human serum albumin (HSA) is the most abundant plasma protein synthesized exclusively in the liver. HSA constitutes over half of the total plasma protein, a concentration of $35-50 \mathrm{~g} / \mathrm{L}$, in a healthy individual. ${ }^{21}$ It is a globular protein consisting of a single peptide chain of 585 amino acids, this globular protein of $66.5 \mathrm{KDa}$ contains 18 tyrosine, 6 methionines, 1 tryptophan, 17 disulfide bridges and 1 free cysteine. It does not contain any carbohydrate or any other non-protein moiety. ${ }^{22}$ It consists of three homologous domains. Each domain can be divided into two subdomains $\mathrm{A}$ and $\mathrm{B}$, which are formed from six and four $\alpha$-helical, respectively. HSA is characterized by high $\alpha$-helical content and a large number of disulfide bonds. Despite very high stability, HSA is a flexible protein with the $3 \mathrm{D}$ structure susceptible to environmental factors such as temperature, $\mathrm{pH}$ etc. ${ }^{21}$

HSA as the major soluble proteins constituent of the circulatory system, it has many physiological and pharmacological functions. One of its main functions is to regulate plasma osmotic pressure between the blood and tissues and it is chiefly responsible for maintenance of blood $\mathrm{pH}$. Another very important role of HSA is that it also functions as a transport molecule. This role is based on albumin, unequal ability to bind a variety of exogenous and endogenous compounds, such as metal cations, fatty acids, amino acids and diverse drug molecules. ${ }^{23}$ HSA is also proposed to serve an antioxidant function in vascular compartment because of its scavenging of reactive oxygen and nitrogen species that are generated by basal aerobic metabolism as well as produced at increased rates during inflammation. ${ }^{24,25}$

HSA is composed of human mercaptalbumin (HMA, reduced form) and non-mercaptalbumin (HNA, oxidized form), that is, a protein redox couple. HMA has one free sulfhydryl group in position 34(Cys-34) and forms the largest fraction of free sulfhydryl in sera. On the other hand, HNA is composed of at least three types of compound and the major HNA compound is a complex of disulfide and cysteine [HNA (Cys)] or glutathione [HNA (Glut)]. The other is an oxidation product with an oxidation number higher than those of mixed disulfides which are extremely small in proportion in extracellular fluids [HNA (Oxi)] ${ }^{26,27}$ An important physiological function of serum albumin is the maintenance of the redox state of the extracellular milieu via the mercapt-nonmercapt conversion. Albumin is known to have a set of diverse beneficial functions, including oncotic pressure regulation and binding and transport capacities for a wide variety of metabolites, including those of therapeutic drugs ${ }^{28,29}$ For this reason, albumin can play an important role in drug disposition and efficacy. ${ }^{30}$ However, the most prominent property of albumin is its major antioxidant activity in a circulatory system that is constantly subjected to powerful oxidative stress. ${ }^{31}$ Previous reports have demonstrated impairment of these properties with the glycation process..$^{32,33}$

\section{Albumin under oxidative stress}

Serum albumin is the most abundant serum protein whose redox modification modulates its physiological function, as well as serves as biomarker of oxidative stress. ${ }^{34}$ In vitro oxidation of amino acid residues leads to protein degradation, aggregation and cross-linking. In constant, significant evidence for the presence of ROS-mediated protein damage in vivo and its possible clinical significance is not currently available. Many studies showed the presence of elevated 
levels of oxidized albumin, in patients with diabetes mellitus type 1 and type 2 ; aging, ${ }^{26}$ patients with chronic hepatitis $\mathrm{C}$, oxidized albumin is a reliable marker of oxidative stress in hemodialysis patients ${ }^{35,36}$ and alteration of redox state of HSA is also seen in patients under anesthesia and invasive surgery ${ }^{37}$ and many other diseases.

In diabetes the circulating albumin level is depressed. A $30-40 \%$ fall in the rate of albumin synthesis in uncontrolled diabetic patients as well as in perfused livers of diabetic can be restored by in vivo insulin; one basis is a marked decline in transcription of albumin mRNA. Albumin degradation and relative extra vascular distribution volume are likewise decreased about $35 \%$ in diabetics. ${ }^{38}$ Other features of diabetes are the effects of non-enzymatic glycation of circulating albumin; two laboratories almost concurrently reported nonenzymatic glycation of serum albumin. ${ }^{39,40}$ Antibodies to the in vivo form of glycoalbumin have been sought but not detected. ${ }^{41}$ If albumin is glycated in the presence of reducing agent such as borohydride, so that the glucose becomes a glucitol-lysine adduct, antibodies to this group are claimed to be widely present in both normal and diabetic subjects, as both $\operatorname{IgG}$ and $\operatorname{IgM}$ isotopes. ${ }^{42}$

\section{Oxygen free radical in diabetes}

The balance between the rate of free radical generation and elimination is important. Excess cellular radical generation can be harmful ${ }^{43}$ however, if there is a significant increase in radical generation, or a decrease in radical elimination from the cell, oxidative cellular stress ensues. ${ }^{44}$ There is convincing experimental and clinical evidence that the generation of reactive oxygen species (ROS) increases in both types of diabetes and that the onset of diabetes is closely associated with oxidative stress. ${ }^{45}$ Oxidative stress plays a central role in the onset of diabetes mellitus as well as in diabetes associated complications. ${ }^{46}$ Various studies have shown that DM is associated with increased formation of free radicals and decrease in antioxidant potential. This leads to oxidative damage of cell components such as proteins, lipids and nucleic acid. In both type 1 and type $2 \mathrm{DM}$ there is increased oxidative stress. ${ }^{47}$

The possible sources of oxidative stress in diabetes might include auto-oxidation of glucose, shifts in redox balances, decreased tissue concentrations of low molecular weight antioxidants, such as reduced glutathione and vitamin $\mathrm{E}$ and impaired activities of antioxidant defence enzymes such as superoxide dismutase and catalase. ${ }^{48}$ ROS generated by high glucose is causally linked to elevated glucose and other metabolic abnormalities important to the development of diabetic complications. However, the exact mechanism by which oxidative stress may contribute to the development of diabetic complications is undetermined. ${ }^{49}$ Hyperglycemia increases the production of reactive oxygen species (ROS) and decreases their scavenging by various mechanisms. Such an increase of ROS may disturb or modify various cellular functions and alter gene expression due to oxidative stress. High glucose induced mitochondrial overproduction of $\operatorname{ROS}^{50}$ and abnormal activation of NAD (P) H oxidase, ${ }^{49}$ are thought to be the major sources of ROS associated with hyperglycemia, while sustained hyperglycemia may also decrease radical scavenging by manganese superoxide dismutase ${ }^{51}$ and the glutathione redox cycle. Thus, chronic oxidative stress is thought to be major cause of diabetic complications and clinical evolution of the accumulation of oxidative stress induced by sustained enhancement of ROS production could be useful to assess the risk of diabetes complications. However, the current clinical markers of ROS in diabetes, such as urinary 8-OH2 -deoxyguanosine, ${ }^{52}$ urinary 8 -iso $\mathrm{PGE}_{2 \alpha}{ }^{53}$ plasma lipid peroxide ${ }^{54}$ and the most important marker oxidized form of HSA [HNA], all reflect short-term ROS formation and not cumulative oxidative stress. Increased oxidative stress in diabetic patients may lead to protein oxidation. The conversion of protein to protein carbonyl (PCO) derivatives occurs via direct oxidation by ROS, with the eventual formation of oxidized amino acids. ${ }^{55}$

Oxidative protein damage cannot be repaired, except for the oxidation of methionine and cysteine. ${ }^{56}$ These oxidations cause irreversible modifications in protein. The structure and activity of oxidized proteins change profoundly in comparison with their native forms. Oxidative modification of proteins in vivo may affect a variety of cellular functions. The best marker for intracellular oxidative stress-dependent cellular damage is the PCO content. The unique advantage of the carbonyl measurement as a good marker of oxidative stress is the fact that it covers a much wider range of oxidative damage than other markers do. ${ }^{57,58}$ The other markers, such as nitro tyrosine, hydroxylation of aromatic and hydrophobic amino acids, are at a very low level in comparison with the carbonyl content. Oxidative stress and more specifically oxidative damage to proteins, is increasingly thought to play a central, mechanistic role in this context as it is associated with modifications in the activities of biological compounds and cellular processes that may be linked to pathological complications. In support, the path physiologic perturbations connected with obesity-related diabetes are robustly associated with hyperglycemia-induced oxidative stress..$^{59,60}$

The aetiology of oxidative stress in diabetes arises from a variety of mechanisms such as excessive oxygen radical production from auto-oxidation of glucose, ${ }^{61}$ glycated proteins and glycation of antioxidative enzymes, which limit their capacity to detoxify oxygen radicals..$^{20}$ In addition to these mechanisms, two others have been suggested as being responsible for the generation of oxygen radicals in diabetes. First, high glucose levels could stimulate cytochrome P450-like activity by excessive nicotinamide adenine dinucleotide phosphate-oxidase (NADPH) produced by glucose metabolism. ${ }^{62}$ Second, ketosis, a hallmark of T1DM in particular, could increase oxygen radical production in diabetic patients. ${ }^{63}$

\section{Conclusion}

The medical significance of oxidative stress has become increasingly recognized and now considered to be a component of virtually every disease process. There exists convincing facts that oxidative stress and reactive oxygen species (ROS) play an important role in the aetiology and/or progression of a number of human diseases. There is substantial evidence that induction of oxidative stress is a key process in the onset of diabetic complications. It has been well documented that HSA is quite vulnerable to reactive oxygen species. This review completes with causal relationship of serum albumin and reactive oxygen species in diabetes very concisely.

\section{Acknowledgements}

None.

\section{Conflict of interest}

The author declares no conflict of interest.

\section{References}

1. Prakash S, Yadav K, Singh JK, et al. Biochemical perspectives of microalbuminuria in diabetes mellitus as early risk markers of nephropathy. Asian Jour of Biomedical Res. 2015;1(3):1-4. 
2. Yadav B, Prakash S, Sah P, et al. Knowledge of Type-II diabetes mellitus and its complications among population of Siraha district, Nepal. Int J of Adv Res. 2016;4(8):19-30.

3. Bluestone JA, Herold K, Eisenbarth G. Genetics, pathogenesis and clinical interventions in type 1 diabetes. Nature. 2010;464(7293):1293-1300.

4. Charonis AS, Reger LA, Dege JE, et al. Laminin alterations after in vitro nonenzymatic glycosylation. Diabetes. 1990;39(7):807-814.

5. Brownlee M. The pathological implications of protein glycation. Clin Invest Med. 1995;18(4):275-281.

6. Cohen MP. Intervention strategies to prevent pathogenetic effects of glycated albumin. Arch Biochem Biophys. 2003;419(1):25-30.

7. West IC. Radicals and oxidative stress in diabetes. Diabet Med. 2000;17(3):171-80.

8. Yadav DP, Prakash S, Sharma S, et al. Biochemical analysis of peroxynitrite modified human serum albumin (PN-HSA) in rheumatoid arthritis and type I diabetes. Ijppr. 2015;4(2):193-206.

9. Griffiths HR. Is the generation of neo-antigenic determinants by free radicals central to the development of autoimmune rheumatoid disease? Autoimmun Rev. 2008;7(7):544-549.

10. Kasper DL, Fauci AS, Longo DL, et al. Harrison's principles of internal medicine (16 ${ }^{\text {th }}$ ed). Neurology. 2005;64(8):1488-1489.

11. Zimmet P, Alberti KGMM, Shaw J. Global and societal implications of the diabetes epidemic. Nature. 2001;414:782-787.

12. Quinn L. Type 2 diabetes: epidemiology, pathophysiology, and diagnosis Nurs Clin North Am. 2001;36(2):175-192.

13. Diagnosis and classification of diabetes mellitus. Diabetes Care 2010;33(Suppl 1):S62-S69.

14. Yadav K, Prakash S. Antimicrobial resistance pattern of uropathogens causing urinary tract infection (UTI) among diabetics. Biomedical Research International. 2016;1:7-15.

15. Roohk HV, Zaidi AR. A review of glycated albumin as an intermediate glycation index for controlling diabetes. J Diabetes Sci Technol. 2008;2(6):1114-1121.

16. Ichikawa H, Nagake Y, Takahashi M, et al. What is the best index of glycemic control in patients with diabetes mellitus on hemodialysis ? Nihon Jinzo Gakkai Shi. 1996;38(7):305-308.

17. Panzer S, Kronik G, Lechner K, et al. Glycosylated hemoglobins (GHb): an index of red cell survival. Blood. 1982;59(6):1348-1350.

18. Yoshiuchi K, Matsuhisa M, Katakami N, et al. Glycated albumin is a better indicator for glucose excursion than glycated hemoglobin in type 1 and type 2 diabetes. Endocr J. 2008;55(3):503-507.

19. Okumura A, Mitamura Y, Namekata K, et al. Glycated albumin induces activation of activator protein-1 in retinal glial cells. Jpn J Ophthalmol. 2007;51(3):236-237

20. Nagayama H, Inaba M, Okabe R, et al. Glycated albumin as an improved indicator of glycemic control in hemodialysis patients with type 2 diabetes based on fasting plasma glucose and oral glucose tolerance test. Biomed Pharmacother. 2009;63(3):236-240.

21. Peters T. All About Albumin: Biochemistry, Genetics and Medical Applications. USA: Academic Press; 1995. 432 p.

22. Carrard G, Bulteau AL, Petropoulos I, et al. Impairment of proteasome structure and function in aging. Int $J$ Biochem Cell Biol. 2002;34(11):1461-1474.
23. Doumas BT, Peters T. Serum and urine albumin: a progress report on their measurement and clinical significance. Clin Chim Acta. 1997;258(1):320

24. Halliwell B, Gutteridge JM. The antioxidants of human extracellular fluids. Arch Biochem Biophys. 1990;280(1):1-8.

25. Halliwell B, Gutteridge JMC. Free Radicals in Biology and Medicine. 2nd ed. Oxford, London, UK: Clarendan Press; 1989. 543 p.

26. Era S, Kuwata K, Imai H, et al. Age-related change in redox state of human serum albumin. Biochim Biophys Acta. 1995;1247(1):12-16.

27. Salgo MG, Bermúdez E, Squadrito GL, et al. Peroxynitrite causes DNA damage and oxidation of thiols in rat thymocytes. Arch Biochem Biophys. 1995;322(2):500-505.

28. Scatchard G, Batchelder AC, Brown A. Chemical, clinical and immunological studies on the products of human plasma fractionation. Vi. The osmotic pressure of plasma and of serum albumin. J Clin Invest. 1994;23(4):458-464.

29. Waldmann TA. Albumin structure, function and uses. In: Rothschild MAE editor. New York, USA: Pergamon Press; 1977.

30. Kratz F. Albumin as a drug carrier: design of prodrugs, drug conjugates and nanoparticles. J Control Release. 2008;132(3):171-183.

31. Halliwell B. Albumin - an important extracellular antioxidant? Biochem Pharmacol. 1988;37(4):569-571.

32. Chesne S, Rondeau P, Armenta S, et al. Effects of oxidative modifications induced by the glycation of bovine serum albumin on its structure and on cultured adipose cells. Biochimie. 2006;88(10):1467-1477.

33. Rondeau P, Singh NR, Caillens H, et al. Oxidative stresses induced by glycated human or bovine serum albumins on human monocytes. Free Radic Biol Med. 2008;45(6):799-812.

34. Fabisiak JP, Sedlov A, Kagan VE. Quantification of oxidative/nitrosative modification of CYS (34) in human serum albumin using a fluorescence-based SDS-PAGE assay. Antioxid Redox Signal. 2002;4(5):855865 .

35. Mera K, Anraku M, Kitamura K, et al. The structure and function of oxidized albumin in hemodialysis patients: Its role in elevated oxidative stress via neutrophil burst. Biochem Biophys Res Commun. 2005;334(4):1322-1328.

36. Soejima A, Matsuzawa N, Hayashi T, et al. Alteration of redox state of human serum albumin before and after hemodialysis. Blood Purif. 2004;22(6):525-529.

37. Hayakawa A, Kuwata K, Era S, et al. Alteration of redox state of human serum albumin in patients under anesthesia and invasive surgery. $J$ Chromatogr B Biomed Sci Appl. 1997;698(1-2):27-33.

38. Murtiashaw MH, Baynes JW, Thorpe SR. Albumin catabolism in diabetic rats. Arch Biochem Biophys. 1983;225(1):256-262.

39. Dolhofer R, Wieland OH. Glycosylation of serum albumin: elevated glycosyl-albumin in diabetic patients. FEBS Lett. 1979;103(2):282-286.

40. Guthrow CE, Morris MA, Day JF, et al. Enhanced nonenzymatic glucosylation of human serum albumin in diabetes mellitus. Proc Natl Acad Sci US A. 1979;76(9):4258-4261.

41. Gregor I, Iberg N, Berger W, et al. Albumin-directed antibodies in diabetes: demonstration of human serum albumin-directed IgM autoantibodies. Diabetologia. 1986;29(8):481-484

42. Mangili R, Viberti GC, Vergani D. Antibodies to human albumin epitopes in type 1 (insulin-dependent) diabetes mellitus. Diabetologia. 1988;31(9):639-646. 
43. Rice EC, Miller N, Paganaga G. Antioxidant properties of phenolic compounds. Tre Pla Sci.1997;2:152-159.

44. Valko M, Leibfritz D, Moncol J, et al. Free radicals and antioxidants in normal physiological functions and human disease. Int $J$ Biochem Cell Biol. 2007;39(1):44-84.

45. Johansen JS, Harris AK, Rychly DJ, et al. Oxidative stress and the use of antioxidants in diabetes: linking basic science to clinical practice. Cardiovasc Diabetol. 2005;4:5.

46. Phillips M, Cataneo RN, Cheema T, et al. Increased breath biomarkers of oxidative stress in diabetes mellitus. Clin Chim Acta. 2004;344(12):189-194.

47. Naziroğlu M, Butterworth PJ. Protective effects of moderate exercise with dietary vitamin $\mathrm{C}$ and $\mathrm{E}$ on blood antioxidative defense mechanism in rats with streptozotocin-induced diabetes. Can J Appl Physiol. 2005;30(2):172-185.

48. Haskins K, Bradley B, Powers K, et al. Oxidative stress in type 1 diabetes. Ann N Y Acad Sci. 2003;1005:43-54.

49. Kowluru RA, Chan PS. Oxidative stress and diabetic retinopathy. Exp Diabetes Res. 2007;2007:43603.

50. Nishikawa T, Edelstein D, Du XL, et al. Normalizing mitochondrial superoxide production blocks three pathways of hyperglycaemic damage. Nature. 2000;404(6779):787-90.

51. Yan H, Harding JJ. Glycation-induced inactivation and loss of antigenicity of catalase and superoxide dismutase. Biochem J. 1997;328 (Pt 2):599-605.

52. Dandona P, Thusu K, Cook S, et al. Oxidative damage to DNA in diabetes mellitus. Lancet. 1996;347(8999):444-445.
53. Patrono C, FitzGerald GA. Isoprostanes: potential markers of oxidant stress in atherothrombotic disease. Arterioscler Thromb Vasc Biol. 1997;17(11):2309-2315.

54. Meagher EA, Barry OP, Burke A, et al. Alcohol-induced generation of lipid peroxidation products in humans. J Clin Invest. 1999;104(6):805-813.

55. Stadtman ER, Levine RL. Protein oxidation. Ann N Y Acad Sci. 2000;899:191-208.

56. Stadtman ER. Importance of individuality in oxidative stress and aging. Free Radic Biol Med. 2002;33(5):97-604.

57. Cakatay U, Telci A, Kayali R, et al. Relation of aging with oxidative protein damage parameters in the rat skeletal muscle. Clin Biochem. 2003;36(1):51-55.

58. Evans P, Lyras L, Halliwell B. Measurement of protein carbonyls in human brain tissue. Methods Enzymol. 1999;300:145-156.

59. Youn JY, Siu KL, Lob HE, et al. Role of vascular oxidative stress in obesity and metabolic syndrome. Diabetes. 2014;63(7):2344-2355.

60. Furukawa S, Fujita T, Shimabukuro M, et al. et al. Increased oxidative stress in obesity and its impact on metabolic syndrome. J Clin Invest. 2004;114(12):1752-1761.

61. Wolff SP. Diabetes mellitus and free radicals: free radicals, transition metals and oxidative stress in the aetiology of diabetes mellitus and complications. Br Med Bull. 1993;49(3):642-662.

62. Jain SK. Hyperglycemia can cause membrane lipid peroxidation and osmotic fragility in human red blood cells. J Biol Chem.1989;264(35):21340 21345.

63. Jain SK, Kannan K, Lim G. Ketosis (acetoacetate) can generate oxygen radicals and cause increased lipid peroxidation and growth inhibition in human endothelial cells. Free Radic Biol Med. 1998;25(9):1083-1088. 